Questions vives

\section{Questions Vives}

Recherches en éducation

$N^{\circ} 28 \mid 2017$

De l'indifférenciation à la différenciation

\title{
L'émancipation sociale, les réseaux des connaissances et les différents fils dont celles-ci sont tissées
}

Inês Barbosa De Oliveira

\section{(2) OpenEdition}

Journals

Édition électronique

URL : http://journals.openedition.org/questionsvives/2713

DOI : $10.4000 / q u e s t i o n s v i v e s .2713$

ISSN : $1775-433 X$

Éditeur

Université Aix-Marseille (AMU)

Édition imprimée

Date de publication : 29 décembre 2017

ISBN : 978-2-912643-52-0

ISSN : 1635-4079

\section{Référence électronique}

Inês Barbosa De Oliveira, «L'émancipation sociale, les réseaux des connaissances et les différents fils dont celles-ci sont tissées », Questions Vives [En ligne], № 28 | 2017, mis en ligne le 15 novembre 2018, consulté le 21 décembre 2020. URL : http://journals.openedition.org/questionsvives/2713;DOI :

https://doi.org/10.4000/questionsvives. 2713

Ce document a été généré automatiquement le 21 décembre 2020.

\section{c)}

Questions Vives est mis à disposition selon les termes de la licence Creative Commons Attribution Pas d'Utilisation Commerciale - Pas de Modification 4.0 International. 


\title{
L'émancipation sociale, les réseaux des connaissances et les différents fils dont celles-ci sont tissées
}

\author{
Inês Barbosa De Oliveira
}

\section{NOTE DE L'AUTEUR}

Version française avec la collaboration de Guilherme João de Freitas Teixeira.

\section{Introduction}

1 Les enjeux pour l'enseignement, pour l'éducation et pour la formation découlant des difficultés actuelles de différencier les savoirs et les valeurs religieuses, d'une part, politiques et scientifiques, de l'autre, sont nombreux. En partant du présupposé selon lequel les différentes connaissances sont inséparables les unes des autres, sans que leurs rapports soient hiérarchiques, mais n'étant surtout pas «la même chose ", nous nous inquiétons très particulièrement de ces confusions, et de leurs dérives au sein de pratiques pédagogiques développées à l'école.

2 Nous nous proposons de réfléchir, dans ce cas, sur la question de comprendre comment les connaissances et les valeurs se constituent pour les sujets sociaux et dans quelle mesure la confusion entre les unes et les autres s'établit au sein de l'école et, d'autre part, comment elle intervient dans les processus éducatifs, mettant en risque leur efficacité. En effet, ce n'est pas une mince affaire d'établir une telle différence et de comprendre comment les connaissances sont tissées en réseaux et sont donc inséparables, et simultanément ont un statut qui leur est propre. Il en va de même pour certaines valeurs éthiques et politiques, fréquemment assumées comme faisant partie des processus éducatifs à l'école mais qui sont confondues assez souvent avec les valeurs religieuses. Ces 
deux défis, ainsi que les difficultés qui en découlent, font partie des exigences posées à une éducation qui se veut émancipatrice.

Des expériences et des récits recueillis lors de notre dernière recherche, développée au long d'une année scolaire, moyennant des activités promues auprès des enseignants du réseau d'enseignement d'une municipalité assez pauvre dans la banlieue de Rio de Janeiro, nous aident à traiter le sujet: notre but était de constituer un corpus de recherche, outre le fait de contribuer à leur formation continue par le dialogue entre les connaissances. La valorisation de pratiques pédagogiques plus démocratiques et fondées sur la solidarité et la non-hiérarchie entre les différentes connaissances - au-delà de la compétition et du privilège des connaissances scientifiques, comprises comme étant la seule vérité - est l'un des objectifs de cette recherche.

$\mathrm{Du}$ point de vue des procédures méthodologiques, toujours en accord avec notre méthodologie de recherche au sein des vécus quotidiens (Oliveira, 2016), nous avons réalisé des rencontres mensuelles qui ont été enregistrées puis étudiées, toujours en quête des indices des pratiques émancipatrices développées par ces enseignants. Ces rencontres ont toujours privilégié la parole de ceux-ci par le biais des conversations et des dialogues entre eux et l'équipe de recherche. Les résultats nous ont permis de trouver certaines pratiques émancipatrices, ainsi que quelques confusions entre différents savoirs et valeurs.

Dans ce texte, nous traitons - à partir de deux récits d'enseignants concernant les sujets susmentionnés - des confusions entre les valeurs de la solidarité et de la charité, ainsi que des risques de confusion entre différentes connaissances lors des démarches de surpassement des hiérarchies scientistes. La tâche d'affronter ces confusions et leurs conséquences ne peut être accomplie que si nous nous adressons aussi bien à l'école et aux espaces de formation qu'à la société et à son organisation sociale qui est aussi à l'origine du problème. Donc, dans ce texte, nous allons présenter quelques concepts et débats sociologiques et politiques majeurs pour la compréhension des enjeux à relever, avant de présenter les récits et leur lecture par rapport à la thématique de l'indifférenciation dans le domaine des savoirs et des valeurs.

\section{La technique et la science comme idéologie}

Déjà en 1968, Habermas $(1973)^{1}$ nous rappelait que la technique et la science devenaient une idéologie sociale dans la contemporanéité, et, par là, mettaient en risque la compréhension de la différence entre science et idéologie, c'est-à-dire entre le domaine de la rationalité cognitive-instrumentale et celui de la rationalité morale-pratique. Le domaine du politique-idéologique, du bon et du mauvais est, donc, envahi par le domaine du vrai et du faux dans cette compréhension du monde ; la confusion qui s'y installe a, peut-être, contribué à la confusion actuelle entre les différentes valeurs éthiques et politiques et le domaine de la vérité scientifique.

7 Ainsi, nous pouvons supposer que le problème reconnu par l'auteur n'était peut-être pas tellement nouveau, mais cinquante ans après il s'est beaucoup éparpillé et approfondi, tout en étant à l'origine_des spécificités entre différents genres - là aussi confondus - de valeurs, celles du domaine du politique et de la vie commune et celles des religions. Les mélanges entre valeurs et connaissances deviennent problématiques un peu partout dans le monde, notamment par le biais de nouveaux courants religieux ou d'idéaux 
conservateurs qui se font passer par des connaissances fondées sur des "vérités " diffusées dans la Bible ou le Coran ou même par des leaders religieux mineurs.

Du côté des valeurs politiques, nous sommes en mesure de reconnaître de plus en plus l'influence des idées et des idéaux religieux dans la politique, comme c'était le cas au Moyen Âge européen. La question qui nous occupe actuellement_est celle de comprendre comment nous avons été ramenés à des débats dans lesquels certains interlocuteurs n'arrivent pas à considérer les différences entre la vie privée des gens et des groupes sociaux avec leurs référents, leurs croyances et leurs modes de vie et la vie publique, le domaine du social et du politique.

Les exemples en sont nombreux, mais nous ne croyons pas qu'il soit utile, pour les objectifs de notre discussion, de les rappeler. Ce qui nous intéresse est plutôt de montrer comment cette confusion et d'autres semblables sont présentes dans le domaine des pratiques scolaires et s'associent à quelques-uns des problèmes vécus par les enseignants et les élèves au sein de la vie quotidienne à l'école. Deux confusions, abordées à partir de deux récits, feront l'objet de ce texte: celle qui se passe entre la valeur politique de la solidarité et la valeur religieuse de la charité, et celle des hiérarchies entre les connaissances. Dans les deux cas, nous croyons que la démocratie et la quête de l'émancipation sociale ont été assez malmenées.

\section{La notion d'émancipation sociale}

Pour Boaventura de Sousa Santos, l'émancipation sociale est :

un ensemble de luttes procédurières sans but défini. Ce qui la distingue des autres jeux de luttes est le sens politique qu'elles assument dans cette processualité. Pour le domaine social, ce sens correspond à l'émancipation, à l'expansion et à l'approfondissement des luttes démocratiques dans tous les domaines structurels de la pratique sociale (1995, p. 277).

De même, pour cet auteur, la démocratie est un système social où les rapports entre les sujets individuels et sociaux, leurs connaissances et leurs modes d'être dans le monde et de le comprendre, s'appuient sur la reconnaissance mutuelle où il n'y a point de place pour les hiérarchies a priori ou fixes.

11 À partir de ces idées, la notion d'émancipation se déplace des sujets vers la société, en les rendant responsables individuellement et collectivement du tissage quotidien et permanent de relations sociales égalitaires et solidaires, de reconnaissance mutuelle, soit des conditions indispensables pour la construction de la justice cognitive et sociale, pour l'émancipation et la démocratie sociales.

12 Étant toujours procédurière et tributaire de la manière dont les défis de la solidarité, de l'écologie entre les savoirs et de la reconnaissance mutuelle sont relevés à chaque instant, cette perspective d'émancipation exige - pour y intervenir - la compréhension des processus quotidiens de tissage de relations sociales et de réseaux de connaissances et de valeurs. Elle exige aussi la reconnaissance de la responsabilité individuelle sur la vie collective, le respect des différentes contributions et des besoins des sujets sociaux. Contribuer à son tissage est aussi une façon de développer des pratiques sociales de justice cognitive, reconnaissant chaque savoir comme une contribution possible au processus social. La validité de chaque connaissance sera définie en fonction de sa contribution possible à la résolution des problèmes collectifs, dans une perspective de complémentarité et d'interdépendance ; il s'établit ainsi une relation plus égalitaire entre 
les sujets de ces différentes connaissances, caractéristique de la justice sociale. D'après Boaventura de Sousa Santos: "L'injustice sociale à l'échelle planétaire est intimement liée à l'injustice cognitive globale; d'où la lutte pour la justice sociale à l'échelle planétaire doit être aussi une lutte pour la justice cognitive globale » (2010, p. 40).

\section{Les réseaux des connaissances et l'écologie entre les savoirs}

13 Si l'émancipation sociale repose sur la création de rapports non hiérarchiques entre les différentes connaissances et cultures - dotées de leurs valeurs, croyances et connaissances -, et par la reconnaissance de l'interdépendance entre elles, le défi démocratique et émancipatoire relève de la lutte pour la reconnaissance de cette interdépendance entre les différents savoirs et de leurs rapports non hiérarchiques. Santos définit cette écologie comme étant « fondée sur la reconnaissance de la pluralité des connaissances hétérogènes (l'une d'entre elles étant la science moderne) et sur des interactions durables et dynamiques entre elles sans pour autant compromettre leur autonomie » (2010, p. 53).

14 Ce défi exige aussi que nous n'oubliions pas que les fils qui se tissent en réseau ont leurs spécificités; autrement dit, l'articulation entre leurs différences ne suppose pas une identité entre eux. Il faut donc reconnaître que chacun possède des spécificités de domaine, de configuration et même de validité.

Contre le scientisme pour lequel il n'y a que les connaissances « scientifiques » qui soient vraies, la notion de tissage des connaissances en réseaux nous aide à voir d'autres contributions et rapports importants. Cependant, il ne faut pas non plus sombrer dans l'idée que tout est toujours valable, parce que cela risque de nous amener aussi bien à un relativisme tétanisant qu'à la confusion entre les domaines des différentes connaissances et leurs applicabilités scientifiques et sociales.

16 Nous assumons ici le slogan des féministes qui affirmaient: «Différents mais pas inégaux. » Apparemment évidente, cette idée est dérangeante pour certaines façons de comprendre les connaissances et leurs processus de production et de légitimation ainsi qu'aux défenseurs des hiérarchies rigides entre les connaissances dites supérieures - du fait d'être considérées comme plus scientifiques - et les autres. L'idée du tissage des connaissances en réseaux nous permet de remettre en question aussi bien la supériorité des certaines connaissances sur d'autres que leur séparabilité dans des domaines propres et indépendants; mais, dans leur inséparabilité, les fils gardent quand même leurs spécificités. Donc, il ne faut pas considérer qu'au cours de ce tissage les fils soient toujours les mêmes, même si l'on pense qu'ils sont reliés entre eux et aux croyances et convictions qui leur donnent du sens (en tant que signification et direction).

Selon la notion de tissage des connaissances en réseaux, celles-ci se tissent en réseaux constitués de tous nos vécus, de tous les modes dont nous nous insérons dans le monde qui nous entoure, et donc n'ayant pas d'origine ni de développement localisables, ni de priorités hiérarchiques, ni de prévisibilité, ni de caractère obligatoire d'itinéraire. Cette idée cherche à dépasser le paradigme de l'arbre de la connaissance, et aussi la façon même dont sont entendus, à cet égard, les processus individuels et collectifs d'apprentissage - cumulatifs et acquis. Concevoir la construction de la connaissance à l'aide de l'image de l'arbre suppose que celle-ci se fait linéairement, par le biais d'un processus où la succession et la séquence des étapes se produisent du plus simple - et basique - au plus complexe, en 
hiérarchisant grâce à une seule opération les connaissances et leurs sujets, selon le

stade de l'arbre où ils se trouvent (Oliveira, 2016, p. 73).

\section{Risques et possibilités de l'écologie des savoirs : des réseaux à la confusion des savoirs}

9 L'expérience que nous avons vécue avec les enseignants nous a montré qu'ils arrivent à bien travailler dans une perspective de l'écologie entre les savoirs, tout en reconnaissant les spécificités propres à chacun ; cependant, il faut prévenir le risque de confondre ces rapports horizontalisés et en réseaux avec un mélange qui puisse rendre identiques les différents savoirs. Ils gardent toujours leur spécificités. Cela existe et devient palpable dans certains discours volontaristes (FREIRE, 1991) sur l'éducation des enfants.

Freire soutenait que l'éducation scolaire ne devrait pas déconsidérer les connaissances antérieures des élèves, et la notion de tissage des connaissances en réseau accompagne ce propos ; toutefois, Freire disait aussi qu'il fallait que l'élève fasse des apprentissages audelà de son acquis. Nous rajoutons que la posture qui se limite au respect des connaissances et des souhaits des enfants est une menace pour la fonction de l'école, qui est de faire apprendre aux enfants des connaissances qui ne font pas partie de leur vie quotidienne.

21 Donc, lors des activités dans lesquelles les connaissances sont reconnues dans leur validité et leur rôle propres dans la solution des problèmes, il ne faut jamais considérer que cet enchevêtrement soit suffisant. Il faut en profiter pour tisser des compréhensions $\mathrm{du}$ monde mieux fondées et formulées que celles qui étaient en place. Pour ce faire, en même temps que la reconnaissance et la promotion des rapports non hiérarchiques entre les savoirs, il faut reconnaître leurs spécificités, les frontières entre eux et les limites des uns et des autres. L'histoire que nous a racontée l'enseignante concernant la promotion de la justice cognitive et de l'écologie des savoirs nous montre son succès dans la première, mais ne nous permet pas de miser sur sa compréhension de la seconde.

Ainsi, ce travail qui visait, même sans les nommer, la promotion de l'écologie des savoirs et la pratique de la justice cognitive - autrement dit, comme proposé par Santos (2010), 
dans le sens de la constitution des rapports non hiérarchiques entre les savoirs, et de la reconnaissance de leur validité -, cherchait à détecter les différentes perceptions des élèves sur la dengue ${ }^{2}$ et les possibilités de l'éviter. Cette enseignante nous a parlé de son expérience avec les élèves du CP :

L'école où je travaille a développé un projet d'étude sur la dengue. Comme mes élèves sont encore en $\mathrm{CP}$, nous avons produit un texte, en faisant la parodie d'une chanson : « C'était une maison ${ }^{3}$ ». Voici notre texte : « C'était une maison tellement bien tenue / où il n'y avait pas d'ordures, ni d'eau stagnante... » Et toute la semaine nous l'avons travaillée en faisant des dessins et des affiches. Les plus réussis ont été amenés à l'exposition pédagogique de l'école ; tout le monde a participé, y compris ceux qui ne savaient pas encore écrire (Enseignante 2).

Il est intéressant de percevoir de son attitude de valorisation de ce que chaque élève savait faire par le biais de ses dessins, en même temps qu'elle commençait à développer un travail avec les plus capables. C'est elle encore qui nous le raconte :

Beaucoup d'entre eux n'arrivaient pas à écrire. Ils ne savaient même pas tenir leurs cahiers, ni leurs crayons! Quelques-uns ne voyaient même pas de quel côté était leur mine. Ils me posaient la question : « Mais il n'y a pas d'encre de ce côté ? » Et je leur montrais le bon côté. Ils souriaient : «Oh, ça marche. » À la fin, ils chantaient notre chanson. Cela n'a pas été facile, mais c'était agréable ! (Enseignante 2).

Pour mieux saisir un projet qui me semblait émancipatoire, j'ai interrogé l'enseignante à propos de la bonne tenue des maisons, en lui demandant si les élèves étaient prêts à partager la responsabilité de supprimer les lieux de ponte et de repos du moustique et s'ils en avaient parlé. En répondant affirmativement, elle nous a expliqué comment cela s'était passé :

Nous avons parlé de la responsabilité de tous concernant la bonne tenue des maisons, parce qu'il ne fallait pas attendre les travailleurs de la santé pour ramasser la bouteille jetée par terre dans l'arrière-cour. Tout le monde peut retourner une casserole. Au lieu de jeter par terre le petit gobelet, on peut le mettre à la poubelle; celle-ci peut être maintenue fermée. Et les enfants apprennent très vite ce genre de choses. Le projet a été développé dans toute l'école. Les travailleurs de la santé sont venus nous rendre visite. Cela a été un projet de travail pour tout le monde, toute l'école y a participé. Les enfants plus âgés ont élaboré des affiches, et ont fait le tour du quartier pour les montrer. Les élèves du CE2 ont préparé un prospectus sur le sujet et l'ont distribué de porte à porte. Ça a été un projet important. Et nous, du CP, comme c'était la première année, nous avons pris à notre compte la partie musicale. Nous avons aussi dessiné le réservoir d'eau avec son couvercle (Enseignante 2).

Une autre enseignante a demandé la parole pour éclairer certains aspects : elle nous a dit que ce détail (dessiner le couvercle des réservoirs d'eau) figurait dans les travaux de quelques élèves; d'après elle, cette attitude nous montre que les élèves connaissent les moyens d'éviter la maladie. Un autre éclaircissement nous a été fourni par la première enseignante :

Ils ont pris soin de bien dessiner, parce qu'on y voit une maison bien entretenue et bien peinte. Il y a juste un arbre: c'est une maison qui empêchera l'entrée du moustique. Ce que je trouve intéressant, c'est qu'il s'agit d'un élève, assez petit, qui a dit: "Ouf, si elle est tellement bien entretenue, il ne faudra pas utiliser des produits, ni brûler des tissus, parce que le moustique n'y arrivera pas, compris?» Alors je lui ai répondu : « Tu as raison, il n'y rentrera pas. » Il a poursuivi : « Voilà, il y a beaucoup de désinfectant pour laisser cette maison bien entretenue. » Alors, j'ai pu m'apercevoir qu'il avait retenu l'idée de ce qui est une maison bien entretenue : le moustique n'a pas la possibilité de se rendre là-bas, parce qu'il n'y a pas de 
saletés ni d'eau stagnante. C'est cela que j'ai trouvé intéressant, puisque cet enfant est l'un des plus petits, il n'était jamais allé à l'école avant. valorisation de la pluralité des connaissances et de la contribution de tous à l'étude et à la prévention contre la dengue, soit une action très claire où la justice cognitive est présente; nous avons donné libre cours à notre curiosité sur ces connaissances en dialogue et en réseau et nous avons voulu connaître l'attitude des familles des élèves. Nous cherchions des informations sur le dialogue de l'école avec d'autres connaissances en dehors de l'école, des familles et des enfants. L'enseignante nous a répondu :

J'ai une classe très fragmentée. Quelques-uns ont la compagnie de leurs parents, d'autres n'en disposent pas. Il y a quatre enfants dont je n'ai jamais vu les familles. $\mathrm{Au}$ dernier jour du projet, destiné à présenter les résultats et en parler publiquement, beaucoup de familles étaient à l'école pour assister à ce que leurs enfants avaient fait. C'est une grande motivation pour les élèves! En fait, il ne s'agit pas que de faire de la peinture, du dessin ou des déguisements en forme de moustique... Il est très important de recevoir les familles dans l'espace scolaire (Enseignante 1).

Des familles aux formats multiples et dont le nombre de personnes est variable, probablement avec des connaissances et des possibilités de dialogue tout aussi multiples. Quel est donc le savoir des familles et des enfants?

Ils savaient déjà presque tout. Ainsi nous avons travaillé sur l'assemblage d'un scénario concernant la dengue. J'ai élaboré des phrases et je les ai mélangées et fixées sur des affiches. Ils ont dû couper et assembler la phrase. Ceux qui savaient déjà lire, ils nous en parlaient: Qu'est-ce qu'on doit faire pour éviter que le moustique de la dengue laisse ses petits œufs là-bas? Ceux qui ont une meilleure connaissance disaient à leur tour : "Il faut que le réservoir d'eau soit toujours couvert. " Ils avaient déjà assez d'informations et échangaient leurs connaissances avec les autres ; ainsi le savoir se diffuse entre eux. Quelques-uns ont l'habitude de regarder les chaînes de télé éducatives. L'un de mes élèves a dit qu'il avait regardé des gens faisant un moustique en pâte à modeler en même temps qu'ils annonçaient ce qu'il faut faire pour éviter ce genre d'insecte chez soi. Alors, comme ils regardent les émissions de ces chaînes-là, ils fournissent ce type de connaissances à la classe. Eux-mêmes, ils ont fait un moustique en pâte à modeler. Il y a même eu un élève qui a dit que le moustique n'arrive à piquer les gens que jusqu'à la hauteur de cinquante centimètres; je le savais déjà, mais ça m'a fait une drôle d'impression qu'un gamin si petit le sache lui aussi (Enseignante 2).

Des connaissances diverses, des familles ayant des profils différents, des élèves ayant différents niveaux d'apprentissage scolaire, des enseignants ayant des performances et des positions plurielles, eux aussi, des classes différentes : une hétérogénéité qui - perçue de façon hiérarchique - ramène à des classements, mais qui, en régime de coopération, dérange le modèle qui exige un seul rythme, le même processus d'apprentissage et le même type de connaissances; en outre, elle peut être perçue comme une interaction productive entre les différentes connaissances, en dialogue les unes avec les autres, un enchevêtrement non hiérarchique, contribuant à la solution des problèmes collectifs concernant la dengue. Cette pluralité et des rapports plus écologiques - d'interdépendance - entre les connaissances apportent une compréhension de la possibilité du tissage de la justice cognitive par le biais des dialogues non hiérarchiques entre les différentes approches et, par là, mènent au tissage de la justice sociale.

Mais il y a également un risque ici : celui de ne pas arriver à distinguer les connaissances les unes des autres, en soustrayant par là leurs spécificités. Connaître une maladie, ses 
modes de transmission et ses symptômes n'est évidemment pas la même chose que la connaître d'un point de vue scientifique. Le mouvement vers la justice cognitive nous aide à ne pas réduire la compréhension du monde à la compréhension scientifique du monde. La différenciation entre les connaissances nous aide à ne pas réduire la compréhension du monde à un mélange dans lequel toutes les interventions sont équivalentes, voire indifférenciées, ce qui peut ouvrir la porte à un retour en arrière vers les superstitions, le miraculeux ou les arguments d'autorité, sans fondement pratique ni théorique. C'est un risque qui ne doit pas être négligé, surtout quand on considère les confusions contemporaines déjà en place dans ces domaines.

\section{De la charité à la solidarité : le religieux et le politique dans les pratiques scolaires}

Concernant la discussion à propos de la confusion entre les valeurs, notre recherche a rencontré l'un des cas emblématiques : quand la notion de charité se confond avec celle de la solidarité, et prend le dessus sur elle. Le défi lancé aux enseignants dans nos cercles de conversation était celui de nous raconter des expériences vécues dans leurs classes au sein desquelles la valeur de la solidarité était présente; par le biais de ce défi, nous voulions les entendre parler des pratiques développées par eux-mêmes avec leurs élèves dans le sens de la formation à la citoyenneté horizontale.

La notion de citoyenneté horizontale est fondée sur le contractualisme rousseauiste et nous la comprenons comme la relation solidaire entre les individus par la reconnaissance de la responsabilité collective sur le bien-être de tous et de chacun. Elle tient compte des éléments du pacte établi entre les individus, en les engageant les uns par rapport aux autres (Silva Júnior, 2006, p. 106). Il s'agit d'une solidarité fondée

sur la conscience d'humanité et d'altérité. Elle ne regarde pas que les intérêts des parties par rapport au tout, mais aussi les intérêts des parties entre elles. L'éveil de la conscience de la citoyenneté ne mène pas qu'à l'exigence du statut civitas de la part de l'individu, mais aussi à la découverte d'un engagement de collaboration afin que tout le monde puisse atteindre ce statut-là (p. 118).

C'est une citoyenneté dotée d'un fort contenu politique ; elle suppose de tenir compte des injustices quotidiennes pour les combattre, en engageant le citoyen eu égard au tissage quotidien de la justice sociale.

Considérant la citoyenneté comme la condition sans laquelle il n'y a guère de démocratie, l'on tire la conclusion qu'elle est active et une activité à mener d'une façon responsable des sujets qui cherchent à créer une société où les lois prônent en faveur de la justice. [...] Le peuple d'où émane le pouvoir n'est pas une masse d'individus désarticulés, mais la collectivité de la citoyenneté, qui suppose l'engagement social et politique (p. 120).

De cette façon, le développement quotidien de pratiques participatives et solidaires dans tous les espacestemps de pratique sociale - y compris dans les écoles et dans les universités - ainsi que la quête d'amplification de leur institutionnalité assument dans ce sens une importance capitale concernant le tissage de l'émancipation sociale. Les pratiques pédagogiques développées à partir de ce point de vue - par leur importance visà-vis de la formation de ceux qui y participent - apparaissent donc comme fondamentales pour leur compréhension.

32 Cependant, lors du questionnement des enseignants à propos de leurs pratiques, axées sur cette citoyenneté, nous nous sommes retrouvée face à des histoires faisant preuve 
d'une idée d'aide à autrui comme charité. Avant même de demander aux enseignants de nous raconter leurs expériences de solidarité, nous avons présenté la notion de citoyenneté horizontale et nous avons proposé une discussion à ce propos :

C'est une idée de citoyenneté que nous commençons à étudier, en la considérant dans la relation des gens les uns avec les autres et non pas des personnes avec l'État. Dans quel sens? Quelle serait alors une relation plus citoyenne entre les gens et de quelle façon l'école peut-elle contribuer à son développement? Notre idée est de comprendre comment les élèves peuvent arriver à construire entre eux des relations de respect, à faire la quête d'un entendement mutuel; et dans quelle mesure, nous les enseignants, nous travaillons à créer des relations qui ont ce profil de recherche d'un entendement mutuel, du respect de l'autre, de la solidarité, et non pas de la charité. C'est bien cela que nous voulons : comprendre comment cette autre idée de citoyenneté nous aide à percevoir les choses qui se produisent à l'école au-delà du programme scolaire (Inês).

Après un moment de silence, normal dans ce genre de situation, les histoires ont commencé à être racontées et discutées. Quelques-unes assez appropriées par rapport à la proposition, et d'autres très concernées par la confusion entre la solidarité et la charité. Pour illustrer la discussion qui nous intéresse, nous allons présenter une histoire de chaque genre.

Une directrice d'école, située dans un quartier très pauvre de la ville, nous a raconté l'initiative collective de faire un " Noël solidaire » : l'achat par les enseignants de cadeaux pour les enfants les plus pauvres de l'école, que les familles n'avaient pas les moyens d'acheter. Les doutes sont apparus au sein même du groupe: quelques institutrices avaient soutenu que leur rôle n'était pas celui de s'occuper de ce genre d'aide caritative aux enfants, même si elles étaient d'accord avec le bienfait de la chose. Ainsi la discussion s'est déroulée entre les défenseurs de cette démarche et les questionnements sur son caractère, solidaire ou caritatif. Au terme de quelques réflexions et débats, tout le monde était presque d'accord sur le caractère plutôt caritatif de la pratique : d'abord parce qu'il était évident que ces enfants se situaient en bas de l'échelle sociale et qu'une aide pareille était conçue comme un soutien « aux plus faibles » offert par les plus forts; et à plus forte raison parce que les défenseurs de la pratique la considéraient comme une démonstration d'amour chrétien, l'obéissance aux consignes de Dieu sur l'obligation d'aider les plus faibles.

L'autre histoire est celle d'Evanildo, âgé de quatorze ans et présentant quelques problèmes mentaux, un élève que l'on considère donc comme «spécial ». Il a été reçu dans une classe de CM1, ce qui a été à l'origine d'un travail de reconnaissance du droit à la différence, du respect mutuel et de solidarité effective. D'après le récit de l'institutrice, les autres enfants de la classe n'ont pas été au courant de son habitude de se quereller, de taper sur ses camarades.

Les enfants ont été très réceptifs et Evanildo n'a plus esquissé de comportements violents. Mais je ne sais pas si c'était à cause de mon discours au cours de l'année : « Nous allons prendre soin d'Evanildo ", « Nous allons nous occuper de lui », « Nous ferons tout pour l'aider "... Les autres enfants ont fini par applaudir tout ce qu'il faisait, ils l'applaudissent... (Enseignante 1).

Ainsi, la commémoration de l'anniversaire d'Evanildo nous permet d'apercevoir comment les élèves ont mis en œuvre la solidarité :

La semaine dernière, nous avons constaté qu'Evanildo avait fêté son anniversaire le jour de la réunion pédagogique. Comme j'ai l'habitude de dessiner un gâteau sur le tableau lors de l'anniversaire de chacun des élèves - outre le fait de chanter «Joyeux anniversaire »-, il m’a demandé de dessiner un gâteau pour lui, parce que 
personne n'avait jamais fait une chose pareille. En fait, n'ayant pas prêté attention à sa date de naissance, la commémoration de celle-ci nous avait échappé. J'ai dit alors aux autres enfants : «Tenez, nous avons oublié l'anniversaire d'Evanildo. » Ensuite, ils se sont organisés à mon insu, et sans demander ma permission, ils m'ont dit qu'ils allaient faire une fête pour Evanildo. J'ai donc décidé d'offrir le gâteau et les enfants se sont occupés des autres choses. [...] J'ai vu un soin particulier de la classe vis-à-vis de ce camarade, qui avait été si mal perçu par moi au début. J'avais tellement peur de recevoir Evanildo dans ma classe! Mais je sens que les autres enfants se font du souci pour lui, ils reconnaissent sa différence et s'en occupent, ils le respectent en tant que camarade, en dépit du fait qu'il est parfois vraiment terrible (Enseignante 1).

Après nous avons encore parlé du comportement d'Evanildo, de la façon dont il agit comme un petit enfant malgré ses quatorze ans à cause de ses problèmes mentaux : il s'agit d'un comportement non souhaitable et imprévu dans les écoles ; cependant, ce n'est pas une bonne raison pour ne pas considérer ses droits à l'éducation, pour ne pas essayer de travailler à partir de ses possibilités. Avec cette idée, l'enseignante cherchait à s'assurer que la classe soit solidaire avec lui, en l'aidant à prendre soin de lui, en le respectant et en le rassurant. La présence d'Evanildo, donc, surgit comme une possibilité de provoquer la pratique de la solidarité dans la classe ; elle permet à l'enseignante de travailler le respect de la différence, la reconnaissance des droits de tous et, surtout, la responsabilité individuelle avec le bien-être de tous et de chacun.

\section{Considérations finales}

Nous voulons réaffirmer notre argument du départ : la confusion entre certains domaines des savoirs et des valeurs pose des problèmes aux processus éducatifs scolaires. Il nous faut donc relever ce défi si l'on assume notre responsabilité vis-à-vis d'une éducation émancipatrice. Les résultats de notre recherche avec les enseignants nous ont permis de mettre en lumière aussi bien les possibilités que les limites et les risques de ce genre de choix pédagogique et de constater la façon dont il est sous l'emprise des questions actuelles concernant la confusion entre les différents domaines symboliques.

Nous partons du présupposé que les connaissances et les valeurs qui sous-tendent notre vie personnelle et collective sont tissées en réseaux, lesquels renferment l'ensemble des connaissances et valeurs différentes qui circulent dans le milieu social où nous vivons et avec lequel nous sommes en interaction. Nous avons aussi considéré que le domaine du religieux, du politique, de l'idéologique, ainsi que celui de l'épistémologique constituent les différentes facettes sous-jacentes à ce que nous sommes, à ce que nous faisons et à ce que nous pensons. Cela dit, il ne faut pas les réduire à leurs fondements, parce que le développement et les fonctionnalités de chacune ont une logique et un fonctionnement particuliers.

Il nous faut donc considérer toujours les réseaux, tout en évitant d'identifier et de confondre les différents domaines des croyances, des valeurs et des connaissances les uns avec les autres sous peine de devenir incapables de proposer des grilles de lecture et des analyses des phénomènes sociaux utiles à la réflexion et à la lutte pour l'émancipation sociale démocratique.

De cette façon, le développement quotidien de pratiques participatives et solidaires dans les écoles ainsi que la quête d'amplification de leur institutionnalité assument une importance capitale concernant le tissage de l'émancipation sociale. Mettre en réseau les 
différentes connaissances visant la solution et le traitement des problèmes réels est aussi important pour le dépassement des hiérarchies entre connaissances et les processus d'exclusion sociale qui en découlent. Les pratiques pédagogiques développées à partir de ces points de vue, par leur importance dans la formation de ceux qui y participent, apparaissent donc comme fondamentales. La démarche consistant à les rendre visibles devient donc essentielle, ainsi que la conscience des risques de créer et d'alimenter des confusions entre valeurs et connaissances mises en place. Il devient aussi impératif de tenir compte de leur pluralité, de leur hétérogénéité et aussi de l'impossibilité de les emprisonner dans les modèles de ce que devraient être les pratiques émancipatrices.

Finalement, la reconnaissance et la valorisation des multiples curricula inventés par les pratiquants (Certeau, 1990) des quotidiens scolaires permettent également de nous éloigner des limites de la pensée moderne - caractérisée par ses hiérarchies entre différentes connaissances et par les valeurs de l'individualisme et de la compétition -, nous plaçant ainsi dans une perspective éducative progressiste et émancipatrice.

\section{BIBLIOGRAPHIE}

Certeau, M. (1990). L'invention du quotidien - 1. Arts de faire. Paris : Gallimard.

Freire, P. (1991). A Pedagogia da esperança. São Paulo : Paz e Terra.

Habermas, J. (1973). La technique et la science comme idéologie. Paris : Gallimard.

Oliveira, I. B. (2016). Le Curriculum, une création quotidienne émancipatrice :l'expérience brésilienne. Paris : L'Harmattan.

Santos, B. S. (1995). Pela mão de Alice. O social e o político na pós-modernidade. São Paulo : Cortez.

Santos, B. S. (2000). A crítica da razão indolente. Contra o desperdício da experiência. São Paulo : Cortez.

Santos, B. S. (2010). Para além do pensamento abissal: das linhas globais a uma ecologia de saberes. Dans B. S. Santos et M. P. Meneses (dir.), Epistemologias do sul (pp. 31-83). São Paulo : Cortez.

Silva Neto, J. L. (2006). Cidadania vertical e horizontal: ensaio para um conceito. Sociedade e direito em revista, 1(1), 105-121.

\section{NOTES}

1. Rédigé en 1968, ce texte n'est apparu en France qu'en 1973.

2. Maladie transmise par le moustique Ædes cegypti, assez répandue à Rio de Janeiro. En matière de prévention collective, la lutte communautaire vise à supprimer les gîtes larvaires à l'intérieur et autour de son habitat: les zones d'eau stagnante comme les réserves d'eau, les vases à fleurs, les dessous de pots, les déchets, les gouttières, etc.

3. Chanson bien connue des enfants brésiliens et très utilisée pour ce genre d'exercice. 


\section{RÉSUMÉS}

Les difficultés actuelles de différencier les savoirs et les valeurs religieuses, d'une part, politiques et scientifiques, de l'autre, sont nombreuses. Ce texte prétend tenir compte de cette question à partir des pratiques pédagogiques développées au Brésil dans le contexte d'une recherche sur les récits d'enseignants à propos de leurs pratiques à caractère émancipateur. L'étude de ces récits a démontré la validité de notre argument du départ : celui selon lequel la confusion entre certains domaines des savoirs et des valeurs pose des problèmes aux processus éducatifs scolaires. En outre, nous avons aussi constaté qu'il nous fallait relever le défi de les travailler si l'on assume notre responsabilité vis-à-vis d'une éducation émancipatrice. Les résultats nous ont également permis de mettre en lumière aussi bien les possibilités que les limites et risques de ce genre de choix pédagogique du fait qu'il est sous l'emprise des questions actuelles concernant la confusion entre les différents domaines symboliques.

The difficulties of differentiating religious knowledge and values from politicians and scientists are many. This text intends to work on this issue from pedagogical practices developed in Brazil in the context of a research on teacher narratives about their emancipatory practices. The study of these narratives has made it possible to make clear our initial argument that the confusion between certain fields of knowledge and values questions school education processes. In addition, we also find that it would be necessary to face the challenge of working with this confusion as we make our commitment to emancipatory education. The results allowed us to highlight the possibilities, limits and risks of this type of pedagogical choice, since these choices are based on current questions regarding the confusion between different symbolic fields.

\section{INDEX}

Mots-clés : éducation et émancipation, justice cognitive, écologie des savoirs, recherche à partir des pratiques quotidiennes

Keywords : education and emancipation, cognitive justice, ecology of knowledge, research from everyday practices

\section{AUTEUR}

\section{INÊS BARBOSA DE OLIVEIRA}

Professeure associée de l'université de l'État de Rio de Janeiro [UERJ] 